\title{
BEYOND RIGIDITY? ESSENTIALIST PREDICATION AND THE RIGIDITY OF GENERAL TERMS
}

\author{
MARIO GóMEZ-TORRENTE \\ Instituto de Investigaciones Filosóficas \\ Universidad Nacional Autónoma de México \\ Departamento de Lógica \\ ICREA and Universidad de Barcelona \\ mariogt@servidor.unam.mx
}

\begin{abstract}
SUMMARY: I offer a brief formal exploration of a certain natural extension of the notion of rigidity to predicates, the notion of an essentialist predicate. I show that, under reasonable assumptions, true "identification sentences" involving essentialist predicates (such as 'Cats are animals') are necessary, and hence that the notion of essentiality is formally analogous in this respect to the notion of singular term rigidity.
\end{abstract}

KEY WORDS: Kripke, Soames, necessity, modal logic

RESUMEN: El artículo hace una breve exploración formal de una extensión natural de la noción de rigidez a los predicados, la noción de predicado esencialista. Muestro que, dados supuestos razonables, las "oraciones de identificación" verdaderas que contienen predicados esencialistas (por ejemplo, 'Los gatos son animales') son necesarias, y por tanto que la noción de esencialidad es formalmente análoga en este sentido a la noción de rigidez para los términos singulares.

PALABRAS CLAVE: Kripke, Soames, necesidad, lógica modal

In his recent book Beyond Rigidity, Scott Soames has forcefully argued that there is no notion that immediately suggests itself as a natural extension of the idea of rigidity to general terms and that satisfies the following two Kripkean conditions: (i) it must apply to typical general terms for natural kinds, stuffs and phenomena, and fail to apply to many other general terms, and (ii) it must be usable in the derivation of the necessitations of true "identification sentences" containing general terms that the extended notion applies to (sentences such as Cats are animals, Water is $\mathrm{H}_{2} \mathrm{O}$ and Lightning is an electrical discharge ought to be examples). In particular, Soames has rejected the conjecture that a certain natural tentative no- 
tion of general term rigidity he himself considers, that of an essentialist predicate, satisfies (i) and (ii). He does not quarrel much with the idea that this notion satisfies (i) (or at least that it satisfies it to an extent compatible with the vagueness of 'typical' and 'many'); but he has argued that it certainly does not satisfy (ii). Space limitations force me to leave for another occasion a discussion of the extent to which the notion of an essentialist predicate satisfies (i), but I will argue here that, under reasonable assumptions, this notion does satisfy condition (ii). More precisely: the notion in question actually gives rise to at least three distinguishable notions of an essentialist predicate, which are analogous to three well-known distinct notions of singular term rigidity; and I will claim that these notions of an essentialist predicate satisfy condition (ii) to the same extent that the analogous notions of rigidity for singular terms satisfy the condition analogous to (ii) in the singular term case.

Before my disagreements with Soames, my numerous agreements. I agree with him about many of the claims he makes in the course of setting up the stage for proper discussion. He recalls that the general terms about which Kripke (1972) makes his key theoretical claims come in a variety of syntactic and semantic categories: they include common nouns (including "mass" common nouns), verbs, and adjectives. If they had all been singular terms or disguised singular terms, then we might have just wondered whether Kripke's notion of rigidity for singular terms applies to them, and if the answer were positive, explore its implications for the question of the necessity of the appropriate "identification sentences", which would then be mere identities. But given that many terms that interest Kripke (e.g., 'cat', 'animal', etc.) are not singular terms, this way of proceeding is not available to the Kripkean.

One possibility is that each general term can be associated in some natural way with a corresponding singular term. Then one might say that a general term is rigid when its associated singular term is rigid and "determine whether these singular terms are rigid. If they are, then true identity sentences in- 
volving them, as well as corresponding theoretical identification sentences containing the original [general terms], will be necessary" (Soames 2002, p. 249). In my opinion, Soames sufficiently discredits the feasibility of options of this type, basically because it is hard to see how to formulate one such option so that not all (or fewer than most) general terms come out rigid (pp. 259-262); notions of rigidity with this defect will fail to satisfy (i) (Soames does not stress that they will also fail to satisfy (ii), because of examples of contingently true "identification sentences" such as Popes are bishops).

Soames makes a reasonably good case that general terms are typically predicative, against views that see many of the grammatically singular ones (such as "mass" nouns like "water', 'lightning' and 'heat') as semantically singular (Soames 2002, pp. 245-248). His main reasons are that the "mass" nouns occur naturally in predicate position (e.g., in That stuff is water), combine with quantifiers to form complex quantifier phrases (Some water) and have bare uses analogous to bare plural uses of predicates (Water is potable). I acknowledge that the claim that general terms are typically predicative deserves fuller discussion; I don't, however, think it's necessary to offer such discussion here. I will simply work under the assumption that the general terms relevant to our discussion are indeed predicative, sometimes availing myself of slightly artificial predicative phrases in the case of "mass" nouns which resist unmodified predicative use in some contexts ("sample of water', 'instance of lightning'); Kripke (1972) often resorts to similar phrases when this suits his purposes ('chunk of gold', 'flash of lightning', etc.). I will be perfectly happy - and probably Kripke would too- if the Kripkean claims can be shown to hold for some notion of general term rigidity which applies to the slightly artificial phrases even if "mass" nouns are "really" semantically singular. I will thus speak from now on simply of "rigid predicates", "predicate rigidity", and so forth.

I also agree with Soames in thinking that, if general terms are predicative, it's natural to view the "identification sentences" 
Kripke is interested in (or many of them, at any rate) as having the form of universally quantified material conditionals or biconditionals; i.e., in symbolic notation, for predicates $A$ and $B$, the sentences

$$
\begin{aligned}
& \forall \boldsymbol{x}(\boldsymbol{A x} \supset \boldsymbol{B x}) ; \\
& \forall \boldsymbol{x}(\boldsymbol{A x} \leftrightarrow \boldsymbol{B} \boldsymbol{x}) .{ }^{1}
\end{aligned}
$$

Kripke too often phrases or rephrases his "identification sentences" in these forms. ${ }^{2}$

Soames says that the key Kripkean doctrine that the necessity of true "identification sentences" involving rigid predicates can be derived from them plus the appropriate rigidity claims - i.e., the Kripkean doctrine that the notion of predicate rigidity satisfies (ii) - can be equated fairly with the thesis that the following basic argument schema, $(\mathrm{P})$, is valid (' $\mathrm{P}$ ' is for 'predicate'): ${ }^{3}$

(Pa) $\forall \boldsymbol{x}(\boldsymbol{A x} \supset \boldsymbol{B x})$ is true $/ \forall \boldsymbol{x}(\boldsymbol{A x} \leftrightarrow \boldsymbol{B} \boldsymbol{x})$ is true;

$(\mathrm{Pb})$ the predicates $A$ and $B$ are rigid;

(Pc) $\forall \boldsymbol{x}(\boldsymbol{A} \boldsymbol{x} \supset \boldsymbol{B} \boldsymbol{x})$ is necessary $/ \forall \boldsymbol{x}(\boldsymbol{A} \boldsymbol{x} \leftrightarrow \boldsymbol{B} \boldsymbol{x})$ is necessary -in other words, $\square \forall \boldsymbol{x}(\boldsymbol{A x} \supset \boldsymbol{B x})$ is true $/ \square \forall \boldsymbol{x}(\boldsymbol{A x} \leftrightarrow \boldsymbol{B} \boldsymbol{x})$ is true.

What the Kripkean needs, then, is to provide a characterization of the notion of predicate rigidity appearing in (claims of the form) $(\mathrm{Pb})$ that makes $(\mathrm{P})$ valid. I think Soames is again more or less right here, but not completely, and this is an important disagreement. As I will note later, a strict analogy with the singular term case ought to lead us to allow that, for some notions of an essentialist predicate, the conclusion of the appropriate argument be something weaker than $(\mathrm{Pc})$.

${ }^{1}$ As in Soames, a text in boldface italics is here an abbreviation for the same text in normal type but flanked by corner quotes.

${ }^{2}$ See Soames 2002, pp. 254-257, for other justifications of the naturalness of this choice.

${ }^{3}$ See Soames 2002, p. 257. 
The intuitive idea of an essentialist predicate is clear enough, but particular ways of making it precise have different implications for the issue that concerns us. Probably the most neutral statement of the idea is made by Soames (2002, p. 251) as follows:

(E) A predicate is essentialist iff the property it expresses is an essential property of anything that has it.

Soames says (2002, p. 251) that the idea behind (E) is expressed by $\left(\mathrm{E}_{W}\right)$ :

$\left(\mathrm{E}_{W}\right)$ A predicate $\mathrm{P}$ is essentialist $t_{W}$ iff for all worlds $w$ and any object $o$, if $\mathrm{P}$ applies to $o$ in $w$, then for all worlds $w^{\prime}$, if $o$ exists in $w^{\prime}$ then $\mathrm{P}$ applies to $o$ in $w^{\prime}$.

$\left(\mathrm{E}_{W}\right)$ is analogous to Kripke's intended characterization of singular term rigidity; ${ }^{4}$ we might call Kripke's notion 'weak rigidity' (and the notion characterized in $\left(\mathrm{E}_{W}\right)$ 'weak essentiality'; it is called simply 'essentiality' by Soames):

$\left(\mathrm{R}_{W}\right)$ A singular term $t$ designating an object $o$ is $\operatorname{rigid}_{W}$ iff for all worlds $w^{\prime}$, if $o$ exists in $w^{\prime}$ then $t$ designates $o$ in $w^{\prime}$ (and $t$ does not designate any object other than $o$ in worlds in which $o$ does not exist).

$\left(\mathrm{E}_{W}\right)$ and $\left(\mathrm{R}_{W}\right)$ being formally analogous, weak essentiality is a natural extension of weak rigidity. Further, at least prima facie, typical predicates for natural kinds, stuffs and phenomena are essentialist $_{W}$, and many other predicates are not. But does weak essentiality satisfy (ii)?

Soames argues that it does not, and his argument is this. $\mathrm{He}$ asks us to make two assumptions, (AQ) and (Res):

(AQ) let us take the range of the universal quantifier at a world to be the domain of objects existing at that world.

${ }^{4}$ For this attribution see especially Kaplan 1989, pp. 569 and 570, n. 8. 
(Res) In talking of predicates, let us restrict ourselves throughout to what is surely the standard case - namely, those that apply to an object at a world only if the object exists at the world. (Soames 2002, p. 257)

(Quantifiers satisfying (AQ), i.e., those whose range at a world is the set of objects existing in that world, are sometimes called 'actualist quantifiers'.) According to Soames, "given these assumptions, what follows from $[(\mathrm{Pa})]$ and $[(\mathrm{Pb})$ with 'rigid' replaced by 'essentialist $\left.W^{\prime}\right]$ is not $[(\mathrm{Pc})]$, but $[(\mathrm{Pd})]$ " (p. 257); in other words, given (AQ) and (Res), argument schema $\left(\mathrm{P}_{W}{ }^{*}\right)$ is invalid even if argument schema $\left(\mathrm{P}_{W} \#\right)$, with the inappropriately weak $(\mathrm{Pd})$ as conclusion, is valid:

(Pa) $\forall \boldsymbol{x}(\boldsymbol{A x} \supset \boldsymbol{B} \boldsymbol{x})$ is true $/ \forall \boldsymbol{x}(\boldsymbol{A} \boldsymbol{x} \leftrightarrow \boldsymbol{B} \boldsymbol{x})$ is true; $\left(\mathrm{Pb}_{W}\right)$ the predicates $A$ and $B$ are essentialist $W$;

(Pc) $\square \forall \boldsymbol{x}(\boldsymbol{A} \boldsymbol{x} \supset \boldsymbol{B} \boldsymbol{x})$ is true $/ \square \forall \boldsymbol{x}(\boldsymbol{A x} \leftrightarrow \boldsymbol{B} \boldsymbol{x})$ is true.

(Pa) $\forall \boldsymbol{x}(\boldsymbol{A} \boldsymbol{x} \supset \boldsymbol{B} \boldsymbol{x})$ is true $/ \forall \boldsymbol{x}(\boldsymbol{A} \boldsymbol{x} \leftrightarrow \boldsymbol{B} \boldsymbol{x})$ is true;

$\left(\mathrm{Pb}_{W}\right)$ the predicates $A$ and $B$ are essentialist $W$;

(Pd) $\forall \boldsymbol{x} \square(\boldsymbol{A x} \supset \boldsymbol{B x})$ is true $/ \forall \boldsymbol{x} \square(\boldsymbol{A x} \leftrightarrow \boldsymbol{B} \boldsymbol{x})$ is true.

This is surely a significant remark, but I think it is at least equally — and perhaps more - significant to point out what happens when one doesn't make assumptions (AQ) and (Res). As it turns out, a wide range of formal logical analogies with the singular term case are preserved if one does not make such highly restrictive assumptions. The existence of these analogies even seems to me to establish that, given the alternative assumption I will make ((PQ) below), the notion of an essentialist predicate does satisfy (ii). But furthermore, in my view, it is at least plausible that Soames's assumption (AQ) is not compulsory for an understanding of typical theoretical "identification sentences" and that his assumption (Res) is not clearly appropriate for a 
descriptive semantical theory of typical terms for natural kinds, stuffs and phenomena. First I will note the formal analogies, and then I will briefly argue that it is plausible to think of Soames's assumptions as descriptively unnecessary or not clearly correct - a less brief discussion of this latter claim will again have to be given elsewhere.

One main reason for considering the notion of weak rigidity is to have a notion that applies to an intuitively rigid singular term independently of what the answer is to the question whether the term designates its object in worlds where that object does not exist. At least for some classes of intuitively rigid singular terms it is not always clear what the answer is, and it is not clear that the answer is not that the term designates its object in worlds where the object does not exist. (At any rate rigid terms for which this is the answer can be introduced into a language by stipulation.) Two natural notions of rigidity stronger than the weak one have also been considered, the first notion applying to a term only if it doesn't designate in worlds where its object does not exist, and the second applying to a term only if it designates its object in all worlds, even in worlds where it doesn't exist. These are the notions of persistent rigidity and obstinate rigidity: ${ }^{5}$

$\left(\mathrm{R}_{P}\right)$ A singular term $t$ designating an object $o$ is $\operatorname{rigid}_{P}$ iff for all worlds $w^{\prime}$, if $o$ exists in $w^{\prime}$ then $t$ designates $o$ in $w^{\prime}$ (and $t$ does not designate any object in worlds in which $o$ does not exist).

$\left(\mathrm{R}_{O}\right)$ A singular term $t$ designating an object $o$ is $\operatorname{rigid}_{O}$ iff for all worlds $w^{\prime}, t$ designates $o$ in $w^{\prime}$.

By analogy, the weak notion of essentiality might and presumably will receive part of its interest from the fact that it applies to an intuitively essentialist predicate independently of what the answer is to the question whether the predicate applies to an object in worlds where that object does not exist (assuming the object to be one to which the predicate definitely applies in

${ }^{5}$ Cf. Salmon 1982, pp. 33-34. 
worlds where it does exist). The answer may not be fully clear in the case of some intuitively essentialist predicates, and for some others it seems reasonable to think that they apply to objects even in worlds in which they don't exist. The predicates 'is an object' and 'is self-identical' are examples of the latter kind (presumably the predicate 'is identical with Plato' is an example too - see below), and we might at any rate create some by stipulation. Further, I will argue later that in the case of typical predicates for natural kinds, stuffs and phenomena it also seems not unreasonable to think that they may apply to objects even in worlds in which they don't exist. Two stronger notions of essentiality suggest themselves by analogy with persistent rigidity and obstinate rigidity:

$\left(\mathrm{E}_{P}\right)$ A predicate $\mathrm{P}$ is essentialist $P$ iff for all worlds $w$ and any object $o$, if $\mathrm{P}$ applies to $o$ in $w$, then for all worlds $w^{\prime}$, if $o$ exists in $w^{\prime}$ then $\mathrm{P}$ applies to $o$ in $w^{\prime}$ (and for all worlds $w^{\prime \prime}$, if $o$ does not exist in $w^{\prime \prime}$ then $\mathrm{P}$ does not apply to $o$ in $\left.w^{\prime \prime}\right)$.

$\left(\mathrm{E}_{O}\right)$ A predicate $\mathrm{P}$ is essentialist $O$ iff for all worlds $w$ and any object $o$, if $\mathrm{P}$ applies to $o$ in $w$, then for all worlds $w^{\prime}, \mathrm{P}$ applies to $o$ in $w^{\prime}$.

It may be noted that Soames's assumption (Res) has the effect that he in fact restricts his attention to essentialist $P$ predicates.

Just as argument schema $(\mathrm{P})$ gives the basic approximate form of the argument that must be validated by a suitably Kripkean notion of predicate rigidity, the analogous basic argument form in the case of singular terms is (ST):

(STa) $\boldsymbol{a}=\boldsymbol{b}$ is true;

$(\mathrm{STb})$ the singular terms $a$ and $b$ are rigid;

(STc) $\boldsymbol{a}=\boldsymbol{b}$ is necessary -in other words, $\square \boldsymbol{a}=\boldsymbol{b}$ is true. 
However, as is well known, under a standard convention for assigning truth-values to atomic statements, this basic form of argument is invalid if we replace 'rigid' in $(\mathrm{STb})$ with either 'rigid $W^{\prime}$ ' or 'rigid $P$ ' —in other words, argument schemata $\left(\mathrm{ST}_{W}{ }^{*}\right)$ and $\left(\mathrm{ST}_{P}{ }^{*}\right)$ are invalid under that standard convention:

(STa) $\boldsymbol{a}=\boldsymbol{b}$ is true;

$\left(\mathrm{STb}_{W}\right)$ the singular terms $a$ and $b$ are $\operatorname{rigid}_{W}$;

(STc) $\square \boldsymbol{a}=\boldsymbol{b}$ is true.

(STa) $\boldsymbol{a}=\boldsymbol{b}$ is true;

$\left(\mathrm{STb}_{P}\right)$ the singular terms $a$ and $b$ are $\operatorname{rigid}_{P}$;

(STc) $\square \boldsymbol{a}=\boldsymbol{b}$ is true.

The reason is that under that standard convention $\boldsymbol{a}=\boldsymbol{b}$ is true in a world if and only if $a$ and $b$ both designate the same object in that world (and hence $\boldsymbol{a}=\boldsymbol{b}$ is false in worlds in which either $a$ or $b$ does not designate).

It is generally agreed, nevertheless, that some versions or modifications of (ST), in which either 'rigid' is replaced with 'rigid $O$ ' or (STc) is suitably weakened, are valid - even under the mentioned standard convention. To be fully explicit, what are commonly agreed to be valid are the following three argument schemata:

(STa) $\boldsymbol{a}=\boldsymbol{b}$ is true;

$\left(\mathrm{STb}_{W}\right)$ the singular terms $a$ and $b$ are $\operatorname{rigid}_{W}$;

$\left(\mathrm{STc}^{\prime}\right) \square($ if a exists, $\boldsymbol{a}=\boldsymbol{b})$ is true. 
(STa) $\boldsymbol{a}=\boldsymbol{b}$ is true;

$\left(\mathrm{STb}_{P}\right)$ the singular terms $a$ and $b$ are $\operatorname{rigid}_{P}$;

$\left(\mathrm{STc}^{\prime}\right) \square($ if a exists, $\boldsymbol{a}=\boldsymbol{b})$ is true.

(STa) $\boldsymbol{a}=\boldsymbol{b}$ is true;

$\left(\mathrm{STb}_{O}\right)$ the singular terms $a$ and $b$ are $\operatorname{rigid}_{O}$;

(STc) $\square \boldsymbol{a}=\boldsymbol{b}$ is true.

Now, if the analogy with the case of singular terms is as close as the Kripkean might hope, we ought to have expected that $\left(\mathrm{P}_{W}{ }^{*}\right)$ is invalid, for the analogous argument schema in the singular term case, $\left(\mathrm{ST}_{W^{*}}\right)$, is also invalid under standard assumptions. But before expanding on this remark, it is important to point out that $\left(\mathrm{P}_{W}{ }^{*}\right)$ is invalid independently of assumption (AQ). Assumptions (AQ) and (Res) are indeed necessary for $\left(\mathrm{P}_{W} \#\right)$ to be valid, but it is important to point out that the assumptions are not needed to show that $\left(\mathrm{P}_{W}{ }^{*}\right)$ is invalid. For $\left(\mathrm{P}_{W^{*}}\right)$ is still invalid if we reject (Res), and it is invalid whether we accept (AQ) or reject it in favor of the most natural alternative, namely the less restrictive (PQ):

(PQ) Let us take the range of the universal quantifier at a world to be the domain of all possible objects, whether they exist or not at that world.

(Quantifiers satisfying (PQ), i.e., those whose range is the set of all possible objects, are sometimes called "possibilist quantifiers'.) That $\left(\mathrm{P}_{W}{ }^{*}\right)$ is still invalid under $(\mathrm{PQ})$ is important because it suggests that (PQ) may be perfectly good if we are in the business of preserving the analogies between the singular term case and the predicate case.

So the appropriate Kripkean claim is that $\left(\mathrm{ST}_{W}\right)$ is valid. But then it is unclear that the mere invalidity of $\left(\mathrm{P}_{W}{ }^{*}\right)$ ought to be seen as a problem for the notion of an essentialist $W_{W}$ predicate. There could only be a problem if "the analogue" 
of $\left(\mathrm{ST}_{W}\right)$ were invalid. But a natural analogue of $\left(\mathrm{ST}_{W}\right)$ in the predicate case, where $(\mathrm{Pc})$ is suitably weakened $\left(\left(\mathrm{P}_{W}\right)\right.$ below), is valid, given assumption (PQ). Let us examine the situation more closely.

Choosing weak rigidity as the preferred notion of rigidity has as a consequence that we get an invalid version of (ST), namely $\left(\mathrm{ST}_{W}{ }^{*}\right)$. But this result does not discredit the notion of weak rigidity nor the thesis of the necessity of true identities involving rigid designators. What it does is to make us note that the counterexamples to $\left(\mathrm{ST}_{W}{ }^{*}\right)$ are of a particular kind, and that they can be deflected by changing (STc) to a weaker but more significant conclusion $\left(\mathrm{STc}^{\prime}\right)$. Specifically, it is easy to see that the counterexamples to $\left(\mathrm{ST}_{W}{ }^{*}\right)$ must all exploit the possibility that a $\operatorname{rigid}_{W}$ term denotes an object in the actual world but fails to denote it in some world in which the object does not exist. Thus, all exploit the consistency with the premises of the hypothesis, uncertain in the case of some intuitively rigid singular terms and incorrect in others, that one is not dealing with a rigid $O$ term. This makes it significant to consider $\left(\mathrm{STc}^{\prime}\right)$, in which the weakening embedded antecedent has the effect of restricting attention exactly to the set of worlds where those counterexamples do not arise.

Similarly, choosing weak essentiality as the preferred notion of an essentialist predicate has as a consequence that we get an invalid version of $(\mathrm{P})$, namely $\left(\mathrm{P}_{W}{ }^{*}\right)$. But this result does not discredit the notion of weak essentiality nor the conjecture of the necessity of true "identification sentences" involving essentialist predicates. What it ought to do is to make us note that the counterexamples to $\left(\mathrm{P}_{W}{ }^{*}\right)$ are of a particular kind, and that they can be deflected by changing $(\mathrm{Pc})$ to a weaker but more significant conclusion. Let's focus on the stronger quantification, $\forall \boldsymbol{x}(\boldsymbol{A} \boldsymbol{x} \leftrightarrow \boldsymbol{B} \boldsymbol{x})$ - always given (PQ), the assumption of possibilist quantification. The following proposition can be easily seen to hold, and helps one to think about the situation ('FC' is for 'form of the counterexamples'):

(FC) Suppose that $\forall \boldsymbol{x}(\boldsymbol{A x} \leftrightarrow \boldsymbol{B} \boldsymbol{x})$ is true in the actual world $r$, and suppose that $A$ and $B$ are essentialist $W$. Then for 
any $w$ and $o$, if $A$ applies to $o$ in $w$ and $B$ fails to apply to $o$ in $w$, either $A$ fails to apply to $o$ in $r$ and so $o$ does not exist in $r$, or $B$ applies to $o$ in $r$ and so $o$ does not exist in $w$. And for any $w$ and $o$, if $B$ applies to $o$ in $w$ and $A$ fails to apply to $o$ in $w$, either $B$ fails to apply to $o$ in $r$ and so $o$ does not exist in $r$, or $A$ applies to $o$ in $r$ and so $o$ does not exist in $w$.

Part of what (FC) states is that every object that serves as a counterexample to the validity of $\left(\mathrm{P}_{W}{ }^{*}\right)$ is an object to which at least one of the predicates involved applies in some worlds (in fact, at least in all worlds in which it exists) but fails to apply in a world in which the object does not exist. Thus all these counterexamples exploit the consistency with the premises of the hypothesis, uncertain in the case of some intuitively essentialist predicates and incorrect in the case of other intuitively essentialist predicates, that one of the predicates $A$ or $B$ is not essentialist $O$. A suitable embedded antecedent which could play the role of $\boldsymbol{a}$ exists in the predicate case ought to work by restricting consideration exactly to the set of worlds in which no such counterexamples can arise. It would single out a significant class of worlds, those in which counterexamples to $\left(\mathrm{P}_{W}{ }^{*}\right)$ could not be generated merely by making model-theoretic choices that for some intuitively essentialist predicates are uncertain and for others incorrect.

Such an embedded antecedent is easy to formulate. (FC) is quite informative, in that it tells us that the counterexamples to the validity of (the second part of) $\left(\mathrm{P}_{W}{ }^{*}\right)$ may be of four kinds: objects that are $A$ 's in a world $w$ but non-existent and non- $A$ 's in $r$, objects that are $B$ 's in $r$ but non-existent and non- $B$ 's in $w$, objects that are $B$ 's in $w$ but non-existent and non- $B$ 's in $r$, and objects that are $A$ 's in $r$ but non-existent and non- $A$ 's in $w$. The embedded antecedent we need ought to exclude precisely the worlds where such objects appear. The embedded antecedent in the second part of $\left(\mathrm{Pc}^{\prime}\right)$ below clearly does the job. (The embedded antecedent in the first part of $\left(\mathrm{Pc}^{\prime}\right)$ of course has half the force.) The corresponding form of argument, $\left(\mathrm{P}_{W}\right)$, is valid under assumption $(\mathrm{PQ})$ : 
(Pa) $\forall \boldsymbol{x}(\boldsymbol{A x} \supset \boldsymbol{B x})$ is true $/ \forall \boldsymbol{x}(\boldsymbol{A} \boldsymbol{x} \leftrightarrow \boldsymbol{B} \boldsymbol{x})$ is true; $\left(\mathrm{Pb}_{W}\right)$ the predicates $A$ and $B$ are essentialist $W$;

$\left(\mathrm{Pc}^{\prime}\right) \square$ (If everything that is actually a B exists and everything that is an $A$ actually exists, $\forall \boldsymbol{x}(\boldsymbol{A x} \supset \boldsymbol{B x}))$ is true $/ \square($ If everything that is actually an $A$ or a $B$ exists and everything that is an $A$ or a $B$ actually exists, $\forall \boldsymbol{x}(\boldsymbol{A x} \leftrightarrow \boldsymbol{B} \boldsymbol{x}))$ is true.

(Also argument schema $\left(\mathrm{P}_{P}\right)$ is valid, in strict analogy with the singular term case:

(Pa) $\forall \boldsymbol{x}(\boldsymbol{A x} \supset \boldsymbol{B} \boldsymbol{x})$ is true $/ \forall \boldsymbol{x}(\boldsymbol{A} \boldsymbol{x} \leftrightarrow \boldsymbol{B} \boldsymbol{x})$ is true;

$\left(\mathrm{Pb}_{P}\right)$ the predicates $A$ and $B$ are essentialist $P$;

$\left(\mathrm{Pc}^{\prime}\right) \square($ If everything that is actually a B exists and everything that is an $A$ actually exists, $\forall \boldsymbol{x}(\boldsymbol{A x} \supset \boldsymbol{B} \boldsymbol{x})$ ) is true $/ \square$ (If everything that is actually an $A$ or $a B$ exists and $e v$ erything that is an $A$ or a $B$ actually exists, $\forall \boldsymbol{x}(\boldsymbol{A x} \leftrightarrow \boldsymbol{B} \boldsymbol{x}))$ is true,

even if argument schema $\left(\mathrm{P}_{P}{ }^{*}\right)$ is invalid, again in strict analogy with the singular term case:

(Pa) $\forall \boldsymbol{x}(\boldsymbol{A x} \supset \boldsymbol{B x})$ is true $/ \forall \boldsymbol{x}(\boldsymbol{A} \boldsymbol{x} \leftrightarrow \boldsymbol{B} \boldsymbol{x})$ is true;

$\left(\mathrm{Pb}_{P}\right)$ the predicates $A$ and $B$ are essentialist $P$;

(Pc) $\square \forall \boldsymbol{x}(\boldsymbol{A x} \supset \boldsymbol{B x})$ is true $/ \square \forall \boldsymbol{x}(\boldsymbol{A x} \leftrightarrow \boldsymbol{B} \boldsymbol{x})$ is true.)

Finally, always given assumption (PQ), argument schema $\left(\mathrm{P}_{O}\right)$ is valid, in analogy with argument schema $\left(\mathrm{ST}_{O}\right)$. As expected, here we don't have any weakening of the conclusion: 
(Pa) $\forall \boldsymbol{x}(\boldsymbol{A x} \supset \boldsymbol{B} \boldsymbol{x})$ is true $/ \forall \boldsymbol{x}(\boldsymbol{A x} \leftrightarrow \boldsymbol{B} \boldsymbol{x})$ is true;

$\left(\mathrm{Pb}_{O}\right)$ the predicates $A$ and $B$ are essentialist $O$;

(Pc) $\square \forall \boldsymbol{x}(\boldsymbol{A x} \supset \boldsymbol{B x})$ is true $/ \square \forall \boldsymbol{x}(\boldsymbol{A x} \leftrightarrow \boldsymbol{B} \boldsymbol{x})$ is true.

It may also be noted that, as is easily seen, $\left(\mathrm{P}_{O}\right)$ is invalid given (AQ). This suggests that (AQ) is not the right assumption if we want some expected analogies between the singular term case and the predicate case to be preserved.

I think all these formal analogies show at least that, given assumption (PQ), the notion of an essentialist predicate satisfies condition (ii) to the same extent that the notion of rigidity satisfies the analogous condition in the singular term case.

But may (PQ) be inappropriate for Kripkean purposes in some respect in which (AQ) is appropriate for them? (PQ) would definitely be inappropriate for Kripkean purposes if some intuitively true "identification sentence" of the kind Kripke had in mind involved typical predicates for natural kinds, stuffs or phenomena and were true given (AQ) but false given (PQ) (the possibility that some such intuitively false "identification sentence" were false given (AQ) but true given (PQ) is excluded merely if we assume that all existents are possible). All existing cats are animals. Is there some non-existing cat that is not an animal? It would seem definitely odd that an object to which the predicate 'cat' applies were an object to which the predicate 'animal' did not apply. To the extent that cases of such objects come up in normal situations, they don't lend support to the inappropriateness of (PQ). We may speak of the unborn cat aborted by my female cat, but then we also speak of it as an unborn animal.

More conclusively, in general (PQ) will not be inappropriate in the respect we are considering provided certain very weak assumptions hold, namely: (1) that the two predicates under consideration are either both essentialist ${ }_{P}$ or both essentialist $O$, and (2) that the "identification sentence" under consideration has the presumable counterfactual force of "hard" scientific claims -in the case of a sentence $\forall \boldsymbol{x}(\boldsymbol{A x} \supset \boldsymbol{B} \boldsymbol{x})$, the force that "if something were to be an $A$, it would be a $B$ " (this claim is 
different from and presumably less controversial than the claim that $\forall \boldsymbol{x}(\boldsymbol{A x} \supset \boldsymbol{B x})$ is true in the actual world when it is literally taken to quantify over all possible objects). ${ }^{6}$ Here is an informal argument: suppose $\forall \boldsymbol{x}(\boldsymbol{A x} \supset \boldsymbol{B} \boldsymbol{x})$ is an intuitively true "identification sentence" involving typical predicates for natural kinds, stuffs or phenomena, which is also true given (AQ); suppose for a contradiction that $\forall \boldsymbol{x}(\boldsymbol{A} \boldsymbol{x} \supset \boldsymbol{B} \boldsymbol{x})$ is false given (PQ); then there is a non-existent object $o$ to which $A$ applies but $B$ doesn't apply; then by (1) $A$ is essentialist $O$, and so $B$ is essentialist $O$ too; but by the (merely weak) essentiality of $A$, if $o$ had existed, $o$ would have been an $A$ and thus, by assumption (2), a $B$; and then $B$ would not be essentialist $O$, a contradiction. ${ }^{7}$

Turning now to the question whether Soames's assumption (Res) is descriptively semantically appropriate, it seems to me that it is by no means clear that it holds for typical predicates for natural kinds, stuffs and phenomena.

One very general consideration in favor of the view that at least many of them are essentialist $O$ is that, unlike some other intuitively essentialist $P$ predicates (like some predicates expressing properties relative to the origin of an object in distinct objects), typical predicates for natural kinds, stuffs and phe-

${ }^{6}$ Some sentences that are asserted in science do not have such counterfactual force, even though they are (for all we know) true if they are explicated as quantified materials or biconditionals with the (AQ) interpretation. But they need not have been the object of Kripke's claims. (Soames 2002, p. 258, gives a taxonomic example to illustrate the invalidity of $\left(\mathrm{P}_{W}{ }^{*}\right)$ given $(\mathrm{AQ})$ : All and only primates are either humans or apes or monkeys or lemurs.) The Kripkean may (and presumably Kripke did) restrict himself to a concern with "identification sentences" taken to support counterfactuals when true. Quantified material conditionals and biconditionals with this property will, if true under the $(A Q)$ interpretation, be true under the (PQ) interpretation. Note that the example based on the sentence Popes are bishops, which I used to argue against an alternative view of predicate rigidity, does not depend on adopting the (AQ) interpretation. That sentence is contingently true given (PQ), as needed for my example, given only the uncontroversial assumption that no non-existent is an actual pope.

${ }^{7}$ Notice that if Soames's assumption (Res) holds of the relevant predicates, then they are all essentialist $P$ and the corresponding true "identification sentences" will be true even given (PQ): they will not be falsified by existing individuals, and they will hold vacuously for non-existents. 
nomena express very basic substantial and intrinsic properties of the objects they apply to. That they are substantial means that they can be mentioned in response to the question What is this object? or What kind of thing is this? Answers like It's a cat, It's an animal, It's a sample of water, It's a sample of $\mathrm{H}_{2} \mathrm{O}$, It's an instance of lightning, It's an electrical discharge seem all appropriate in the relevant cases. That they are intrinsic means that an object does not possess them in virtue of being in a relation to some other distinct objects (which therefore need not exist in order for the object to possess the properties we are talking about). It is prima facie plausible to think that the substantial intrinsic properties of an object are so basic to its identity that we will be tacitly inclined to see them as applying to their objects even in worlds in which these objects do not exist.

Further, I think a number of examples from natural language suggest that at least many of the typical predicates for natural kinds, stuffs and phenomena may be best treated as


itively true sentences which, under standard semantic assumptions, are true only if the predicates of the relevant sort that they contain apply in a world to some objects not existing in that world. Since in this particular case there seems to be no reason to think that any object or world should receive special treatment, the examples suggest that those predicates apply in all worlds to all objects they apply to in some world, and hence that those predicates are essentialist ${ }_{O}$.

Let's consider some of the examples. ${ }^{8}$ They are inspired by familiar examples with the help of which it has been argued that proper names are $\operatorname{rigid}_{O}$. Some of these examples exploit the analogy between tense and modality, as in Plato is dead, which seems true now, but which under standard semantic as-

${ }^{8}$ In fact, we already have one. We spoke naturally of the unborn cat aborted by my female cat. With the help of this description we may utter, for example, a sentence like The unborn cat aborted by my female cat is one of the many animals that remain unborn. This is intuitively true, but under standard semantic assumptions it is true only if some non-existing object is a cat and many non-existing objects are animals (assuming that a cat does not exist until born). 
sumptions is true at a time $t$ only if 'Plato' designates at $t$ something (and what else than Plato?) that doesn't exist at $t$. Without exploiting the analogy, we may consider a sentence like Plato was prevented from developing when sperm $\mathrm{S}$ and egg $\mathrm{E}$ did not unite and went on to die, which is presumably true in some possible worlds (assume ' $S$ ' and ' $E$ ' to be names of the sperm and egg from which Plato actually developed), but which is true in a possible world $w$ only if 'Plato' designates in $w$ an object (and which if not Plato?) which does not exist in $w$.

Analogously, we may consider the sentences Some men (human beings) are dead and Some men (human beings) were prevented from developing when sperm $\mathrm{S}_{1}$ and egg $\mathrm{E}_{1}$, and sperm $\mathrm{S}_{2}$ and egg $\mathrm{E}_{2}$, did not unite and went on to die. The first would seem true now, but under standard assumptions it is true at a time $t$ only if some object that is a man at $t$ does not exist at $t$. The second would seem true (given suitable stipulations about the names it contains), even in the actual world, but under standard assumptions it is true at a world $w$ only if some object that is a man at $w$ does not exist at $w$.

Similar examples can be constructed with other typical terms for natural kinds, stuffs and phenomena. Consider, for example, the sentence Some instances of lightning were prevented from arising when the wind separated charged clouds $\mathrm{C}_{1}$ and $\mathrm{C}_{2}$. This is intuitively true (given suitable stipulations about the names it contains), and on the usual view it is true at a world $w$ only if some object that is an instance of lightning at $w$ does not exist at $w$.

Appropriately, many other predicates clearly do not give rise to intuitively true sentences when they are substituted for the predicates we have used in the examples, even if they apply to their objects in some worlds in which they come into existence -in fact, even if they apply to them in all worlds in which they come into existence, and are therefore essentialist $t_{P}$. The following sentences all seem clearly false:

Some things that climbed Everest were prevented from developing when sperm $S_{1}$ and egg $E_{1}$, and sperm $S_{2}$ and egg $E_{2}$, did not unite and went on to die. 
Some things people saw in the sky were prevented from arising when the wind separated charged clouds $C_{1}$ and $C_{2}$.

Some things that developed from sperm and egg cells were prevented from developing when sperm $S_{1}$ and egg $E_{1}$, and sperm $S_{2}$ and egg $E_{2}$ did not unite and went on to die.

There are, to be sure, other theoretical ways of accounting for the intuitions about the truth-value of these examples than to postulate that 'man', 'instance of lightning', etc., are essentialist $_{O}$ - just as there are other theoretical ways of accounting for the intuitions about the truth-value of Plato is dead and of Plato was prevented from developing when sperm $\mathrm{S}$ and egg $\mathrm{E}$ did not unite and went on to die (in some worlds) than to postulate that 'Plato' is $\operatorname{rigid}_{O}$. As I warned, a more extended discussion of this topic will have to be given elsewhere. Nevertheless, even before such discussion can be given, the examples have the value of illustrating how pretheoretical intuitions do not uncontroversially support Soames's assumption (Res) in the case of typical predicates for natural kinds, stuffs, and phenomena.

\section{REFERENCES}

Kaplan, D., 1989, “Afterthoughts”, in J. Almog, J. Perry and H. Wettstein (eds.), Themes from Kaplan, Oxford University Press, New York, pp. 565-614.

Kripke, S., 1972, "Naming and Necessity", in D. Davidson and G. Harman (eds.), Semantics of Natural Language, Reidel, Dordrecht, pp. 253-355, 763-769. Book edition with an added preface: Naming and Necessity, Harvard University Press, Cambridge, Mass., 1980. (References to the book edition.)

Salmon, N., 1982, Reference and Essence, Blackwell, Oxford.

Soames, S., 2002, Beyond Rigidity, Oxford University Press, New York.

Received July 5, 2004; accepted October 6, 2004. 